\title{
Design of an ECG sensor circuitry for cardiovascular disease diagnosis
}

\begin{abstract}
ECG biosignal conditioning $(\mathrm{BC})$ is critical because it directly affects measurement accuracy, reliability, and repeatability. It also presents a great challenge due to the small amplitude of ECG raw signals and their ease of corruption with noise and other disturbances. This paper describes how an ECG biosignal conditioning circuitry was designed, built, and tested. The circuit consists of an instrument amplifier (AD8220), a 1st-order active high-pass filter (containing a MCP6271 op-amp and a RC filter in Sallen-Key configuration), a 5th-order active Bessel low-pass filter (consisting of a 1st-order LPF and two 2nd-order Sallen-Key filters), and a Twin-T active notch filter (combining two " $\mathrm{T}$ " shape RC filters with a MCP6271 op-amp). A right leg drive circuit was added in order to cancel the common-mode signal between the left and right arm electrodes. A power supply circuit provides a $\pm 5 \mathrm{~V}$ DC source for the system using two $9 \mathrm{~V}$ batteries and two voltage regulators (NTE977 regulates $+9 \mathrm{~V}$ power supply to +5 V; NTE1917 regulates $-9 \mathrm{~V}$ supply to $-5 \mathrm{~V}$ ). Data acquisition and sampling were performed using a USB6009 module with a built-in A/D converter. Testing of a real electrocardiogram from a human subject was performed on the designed $\mathrm{BC}$ circuit, which has indicated that the developed BC circuitry can preserve useful ECG information while removing unwanted noise and interference components.
\end{abstract}

Volume 2 Issue 4 - 2017

\author{
Winncy Y DU,Winston JOSE \\ Mechanical Engineering Department, San Jose State University, \\ USA
}

Correspondence: Winncy Y DU, Mechanical Engineering Department, San Jose State University, San Jose, California 95 I I6, USA, Tel + I-408-924-3866, Fax + I-408-924-3995, Email winncy.du@sjsu.edu

Received: April 22, 2017| Published: May 02, 2017

Keywords: biosignal conditioning, ECG, passive and active filters

\section{Introduction}

Sensors have been playing key roles in all aspects of health care, especially Cardiovascular disease (CVD) -the disease that is the No.1 killer in the United States and the single leading disease that ended 17.3 million people's lives worldwide in $2013 .{ }^{1}$ Unlike many other diseases, some CVDs can cause sudden death, especially in people who physically appear heathy and fit. Early diagnosis and warning, therefore, are extremely important to uncover hidden or silent heart diseases and prevent sudden death. In addition, the cost for detecting and treating cardiovascular disease is very high, thus early diagnosis and prevention post a significant financial benefits as well. Sensing and monitoring technologies for heart deceases have attracted great attention from health care, engineering, industry and information processing societies.

The sensor technology and development for CVD studies and diagnosis mainly focus on
i. Detect heart pulse waveforms (e.g., ECG, TCPD);
ii. Measure blood properties (e.g., pressure, viscosity, contents and their percentage);
iii. Create heart or vessel images (e.g., CT, MRI, Ultrasonic); and
iv. Establish wireless sensor network.

The electrocardiogram (EKG or ECG) is a graph that records the electrical activity of the heart. Its amplitude has a range of $0.1 \mathrm{mV}$ $10 \mathrm{mV}$; while its frequency falls into $0.01 \mathrm{~Hz}-250 \mathrm{~Hz}$. ECG is an important tool used for support of diagnosis and treatment of various cardiac and other related diseases:

Be the first indicator of ischemia and help in proper use of thrombolysis in treating ischemia. i. Help in diagnosis of the cause of the chest pain.

ii. Help in diagnosis of the cause of the dyspnea.

iii. Help in diagnosis of electrolyte disturbances.

iv. Help in diagnosis of adverse drug effects.

v. Help in diagnosis of structural changes of the heart.

vi. Identify a potentially life-threatening arrhythmia.

vii. Pinpoint the chronic effects of sustained hypertension or the acute effects of a massive pulmonary embolus.

An ECG is usually obtained by means of metal electrodes placed on the surface of the body. However, the raw ECG signals directly from the electrodes are inherently weak $(0.001 \mathrm{mV}-100 \mathrm{mV}$ with a typical value of $1 \mathrm{mV}$. They are easily corrupted with noise and other disturbances such as power line interference, impulse noise, electrostatic potentials, stray capacitance, and nearby electronic devices. Biosignal artifacts can also be introduced into an ECG by subject movement and muscle tension. ${ }^{2}$ All of these facts have posted a great challenge on ECG data. The biosignal conditioning (BC), therefore, has played a key role in ECG and many other biomedical instruments and biosensors. A well designed BC circuit can significantly improve measurement accuracy, reliability, and repeatability.

Several works on ECG sensing have been reported in the literature. ${ }^{3-12}$ Tenedero et al. $^{3}$ developed an ECG circuit with a bandwidth of $0.05 \mathrm{~Hz}-40 \mathrm{~Hz}$. An AD620 instrumentation amplifier (IA) was employed due to its low noise, low input bias current, low offset voltage, low power, and high Common Mode Rejection Ratio (CMRR) of $100 \mathrm{~dB} .^{3}$ Three filters were used between the IA and the data acquisition unit: an isolation amplifier (isolates $60 \mathrm{~Hz}$ power line and also protects the patient from cardiac shock), a high-pass filter 
with a cutoff frequency of $0.05 \mathrm{~Hz}$, and a low-pass filter with a cutoff frequency of nearly $100 \mathrm{~Hz}$. The sampling rate in the ADC (analog to digital conversion) for the ECG signal is $500 \mathrm{~Hz}$. Fulford-Jones et al. ${ }^{4}$ designed a portable, low-power ECG system. ${ }^{4}$ An operational amplifier (op-amp) embedded in a single INA321 chip was used due to its low noise and low power consumption. This op-am has a CMRR of $94 \mathrm{~dB}$. A high-pass feedback filter corrects any DC shift occurring over time. Their ADC sampling rate is $120 \mathrm{~Hz}$. Matviyenko ${ }^{5}$ used a CY8C27443 microcontroller for ECG signal acquisition and processing. The IA embedded in the controller has a CMRR of $60 \mathrm{~dB}$ According to the author, this low CMRR was acceptable due to the unique design which placed a differential low-pass filter before the IA to reduce radio frequency interference (RFI), because RFI error cannot be filtered out after the ECG signal has been rectified by the IA. A $2 \mathrm{kHz}$ cutoff frequency high-pass filter was placed at the output of the IA. A buffer amplifier and an inverting amplifier were also used to cancel the RFI interference. The ADC sampling rate is $240 \mathrm{~Hz}$. Two industry leaders, Texas Instruments (TI) ${ }^{6}$ and Analog Devices Inc. (AD) ${ }^{7}$ also developed the ECG conditioning circuits. TI's circuitry features an INA321 IA with several unique features: a power down mode that shuts down the circuit when the supplied current is less than $1 \mathrm{~mA}$ (for power saving), embedded op-amps in the microcontroller, a feedback loop that maintains a constant DC level, and a $512 \mathrm{~Hz}$ sampling rate. Further filtering was digitally implemented to remove power line noise and to provide a pass band of $6 \mathrm{~Hz}-30 \mathrm{~Hz}$. In AD's design, the ECG circuit uses AD's AduC842 (an integrated "system on chip") to perform amplification, digital filtering, and A/D conversion. This paper discusses the design of an electrocardiogram (ECG) sensor circuitry for cardiovascular disease diagnosis. The paper starts with obtaining the real ECG signals (Section II), followed by signal conditioning circuit design and implementation (Section III). ECG Signal testing and results using the designed $\mathrm{BC}$ circuit are described and shown in Section IV. Finally Section V draws the conclusions.

\section{Obtaining an real ECG signal}

An Electrocardiogram (ECG) is usually recorded by electrodes placed at specific points on the body. The electrode used for recording the ECG signal is silver-silver chloride (Ag- $\mathrm{AgCl})$. It has the following important features: (1) it is non-polarizable, meaning that current flows freely across the electrode junction. No electrons accumulate in the junction as in a polarizable electrode; (2) it generates less noise $(<10 \mu \mathrm{V})$. The $\mathrm{Ag}-\mathrm{AgCl}$ electrode has an $\mathrm{AgCl}$ layer deposited on an Ag plate. The $\mathrm{Cl}^{-}$ions move in the human body (in the electrolyte). In the $\mathrm{AgCl}$ layer, these $\mathrm{Cl}^{-}$ions are converted to electron flow in the $\mathrm{Ag}$ plate and these electrons are then sent out through a connecting wire. This $\mathrm{Ag}-\mathrm{AgCl}$ structure reduces the $\mathrm{DC}$ offset potential to a very small value. A conductive gel is used to minimize the disturbance of the double charge layer. Three limb leads are commonly used to construct an Einthoven's triangle (Figure 1). ${ }^{13}$

An ECG wave can be recorded by placing leads at electrically equidistant points on the body from the heart, thus maximizing the potential difference between leads. ${ }^{14}$ Lead I is configured as the positive electrode on the left arm $(L)$, the negative electrode on the right arm $(R)$; Lead II is configured as the positive electrode on the left leg/foot $(F)$, the negative electrode on the right arm $(R)$; Lead III sets the positive electrode on the left leg/foot $(F)$, the negative electrode on the left arm $(L) . V_{I}, V_{I I}$, and $V_{I I I}$ denote the voltage of Lead I, Lead II, and Lead III, respectively. $\Phi_{L},{ }_{R}$, and $\Phi_{F}$ are the voltage potentials at the points $L, R$, and $F$, respectively. The Cardiac Vector's magnitude $|\bar{P}|$ and direction $\alpha$ can be expressed by. ${ }^{14}$

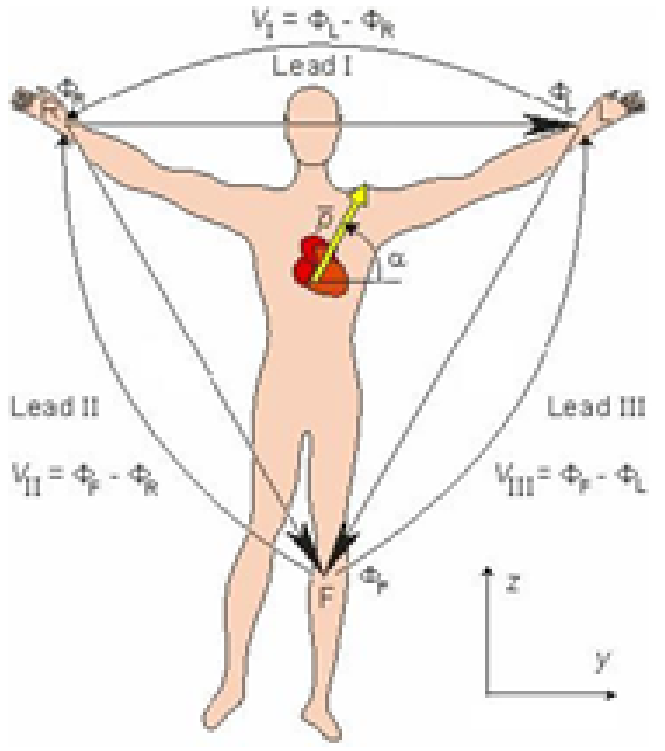

Figure I Einthoven's triangle and limb lead configuration. ${ }^{13}$

$$
\begin{aligned}
& |\bar{P}|=2 \sqrt{\left(V_{I}^{2}+V_{I I}^{2}-V_{I} V_{I I}\right) / 3} \\
& \text { or }|\bar{P}|=2 \sqrt{\left(V_{I}^{2}+V_{I I I}^{2}+V_{I} V_{I I I}\right) / 3} \\
& \alpha=\tan ^{-1}\left(\left(V_{I}-2 V_{I I}\right) /\left(\sqrt{3} V_{I}\right)\right) \\
& \text { or } \alpha=\tan ^{-1}\left(\left(V_{I I}+V_{I I I}\right) /\left(-\sqrt{3} V_{I}\right)\right)
\end{aligned}
$$

The typical ECG signal obtained from the $\mathrm{Ag}-\mathrm{AgCl}$ electrodes is $1 \mathrm{mV}$ in amplitude and it is easily corrupted by noise. The main sources of noise include respiration, motion artifacts, muscle contraction, electrode contact noise, power line interference, RFI, and electromagnetic interference (EMI). In certain situations, noise can completely override the ECG waves and make the amplified signal useless.

\section{Signal conditioning circuit design and implementation}

To effectively remove unwanted noise and preserve the useful components of ECG signals, the following biosignal conditioning schemes and sequence were developed:

i. Amplify the raw ECG signal with an instrumentation amplifier to raise the signal voltage level.

ii. Use a high-pass filter to eliminate DC offset developed between electrodes.

iii. Apply a low-pass filter to remove high frequency noise.

iv. Filter out power line interference using a notch filter.

v. Convert the filtered analog ECG signal to digital for computer display and/or further digital signal processing and analyses.

A block diagram of the $\mathrm{BC}$ circuit is shown in Figure 2. The following sections will discuss component functions and features.

\section{AD8220 instrumentation amplifier}

An Analog Devices' AD8220 instrumentation amplifier (IA) was 
used first to amplify the ECG signal obtained from the potential difference between the right and left arm electrodes (Lead I in Figure 1). The AD8220 has a wide operating range in noisy environments, low input bias current of $10 \mathrm{pA}$, high CMRR with little effect from RFI, and adjustable gain. ${ }^{15}$ Since the voltage source $V_{s}$ of the AD 8220 is $\pm 5 \mathrm{~V}$, the gain $G$ was conservatively set at 19 to avoid both output voltage saturation, although a higher gain is achievable with this IA. At this setting, a typical $1.0 \mathrm{mV}$ ECG signal is amplified to $19.0 \mathrm{mV}$ -well below the $V_{s}$ level. The gain resistor $R_{\mathrm{g}}$ is calculated by. ${ }^{16}$

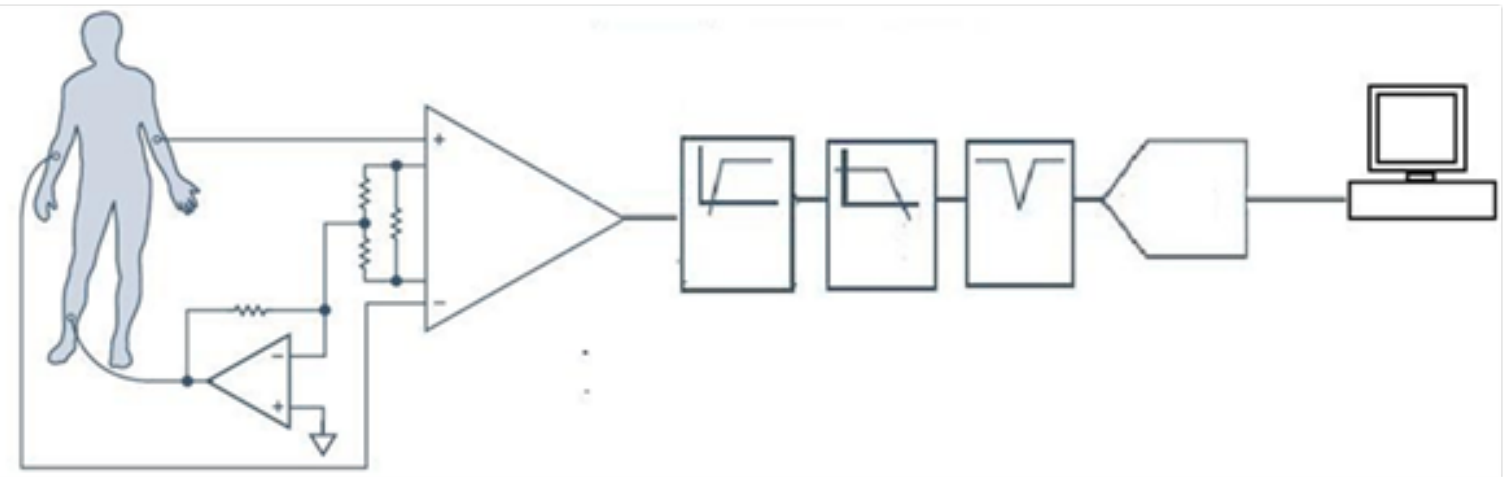

Figure 2 Overall block diagram of the ECG conditioning circuit.

$$
R_{g}=\frac{49.4 \mathrm{k} \Omega}{G-1}=\frac{49.4 \mathrm{k} \Omega}{19-1}=2.74 \mathrm{k} \Omega
$$

\section{First-order active High-pass Filter (HPF)}

After the amplification, the ECG signal passes through a noninverting active high-pass filter (HPF) to eliminate DC offset (developed between the electrodes) and to be amplified further. This active HPF consists of a MCP6271 op-amp, a RC filter (capacitor $Z_{2}$ and resistor $Z_{4}$ ) and the gain resistors $Z_{10}$ and $Z_{11}$ arranged in a SallenKey configuration (Figure 3). The bandwidth of the ECG recording system is very important in order to achieve an accurate recording of cardiac signals. The American Heart Association (AHA) recommends a minimum bandwidth of $150 \mathrm{~Hz}$ for children between the ages of 12 to 16 years; and a minimum bandwidth of $125 \mathrm{~Hz}$ for adults. ${ }^{17}$

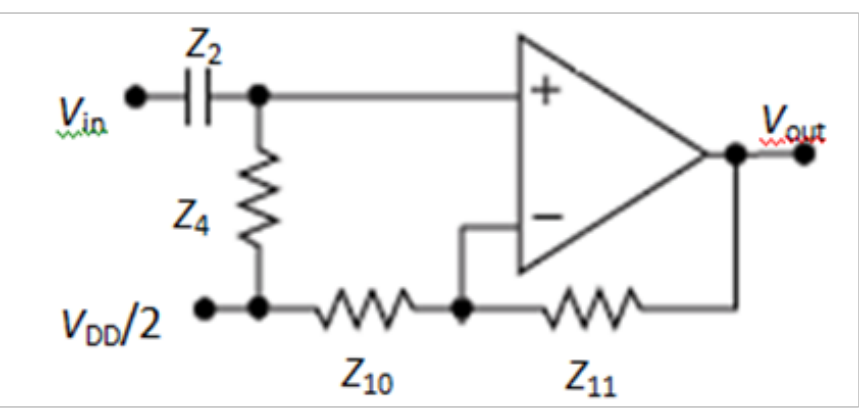

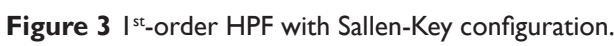

Based on these recommendations, the ECG signal was set to a frequency range (bandwidth) of $0.01-100 \mathrm{~Hz}$. To ensure the most useful ECG signals pass through the HPF, a cutoff frequency $f_{\mathrm{c}}$ of $0.033 \mathrm{~Hz}$, instead of $0.01 \mathrm{~Hz}$, was chosen in consideration of manufacturing tolerances for electronic components. $Z$, was set to $6.8 \mu \mathrm{F}$ for a faster response. Thus, the resistor $Z_{4}$ has a value of:

$$
Z_{4}=\frac{1}{2 \pi Z_{2} f_{c}}=\frac{1}{2 \pi\left(6.8 \times 10^{-6}\right)(0.033)}=710 \mathrm{k} \Omega
$$

In addition, $Z_{10}$ and $Z_{11}$ were set to $806 \Omega$ and $13 \mathrm{k} \Omega$ respectively. The resulting HPF gain, $G_{\mathrm{HPF}}$, is:

$$
G_{H P F}=1+\frac{Z_{11}}{Z_{10}}=1+\frac{13000}{806}=17.13
$$

\section{Fifth-order active bessel low-pass filter}

After the HPF, the ECG signal is sent to a low-pass filter (LPF) to remove its high frequency noise components. $160 \mathrm{~Hz}$ was chosen as the cutoff frequency based on the normal ECG frequency range as well as the AHA guidelines for minimum ECG frequency bandwidth. The LPF is a cascaded 5th-order active Bessel filter which has excellent transient and linear phase response. ${ }^{18}$ In comparison, other filter types, like the Chebychev, have overshoot (ripple) in their passband magnitude response and transient response. An active filter kit from Microchip Technology was employed for the filter setup. A total of three active filters were needed to form this 5th-order Bessel filter (Figure 4).

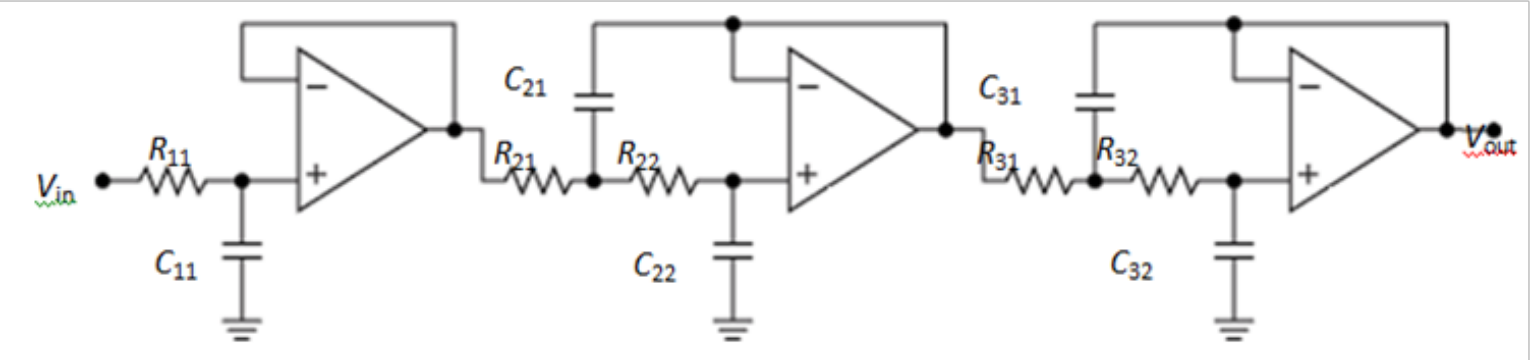

Figure 4 Circuit diagram of the 5th-order active Bessel LPF. 
The first filter is an active 1st-order LPF which has a real pole in its transfer function; the second and the third filters both have 2ndorder Sallen-Key topologies (each has a pair of complex conjugate poles in their transfer function). All three filters use unity gain (the Sallen-Key topology inherently has excellent gain accuracy as a unity-gain buffer). The unity gain configuration also requires less components-two resistors versus four resistors in non unity-gain designs, thus reducing thermal noise caused by resistors. The resistors and capacitors in the LPF were determined as follows, considering Filter 2 first:

I. Set $f_{c}=160 \mathrm{~Hz}$ :

$$
f_{c}=\frac{1}{2 \pi \sqrt{R_{21} R_{22} C_{21} C_{22}}}=160 \mathrm{~Hz}
$$

II. Let $m$ be the ratio of $R_{21}$ and $R_{22}, n$ the ratio of $C_{21}$ and $C_{22}$, i.e.:

$$
\left\{\begin{array} { l } 
{ R _ { 2 1 } = m R } \\
{ R _ { 2 2 } = R }
\end{array} \left\{\begin{array}{l}
C_{21}=n C \\
C_{22}=C
\end{array}\right.\right.
$$

III. Let Q-factor $=1$ (for better filter performance at cutoff frequency without significant overshoot), i.e.,

$$
Q=1=\frac{\sqrt{R_{21} R_{22} C_{21} C_{22}}}{C_{22}\left(R_{21}+R_{22}\right)}=\frac{\sqrt{m n}}{m+1}
$$

IV. Choose $m$ and determine $n$ based on $Q$ setting,

If $m=1$, then from (10) $n=4$.

\section{Select $C$ and calculate $R$ based on $f_{c}$ :}

$C$ should not be too small since low capacitor values can result in significant errors due to parasitic capacitance. Chose $C=0.068 \mu \mathrm{F}$, thus:
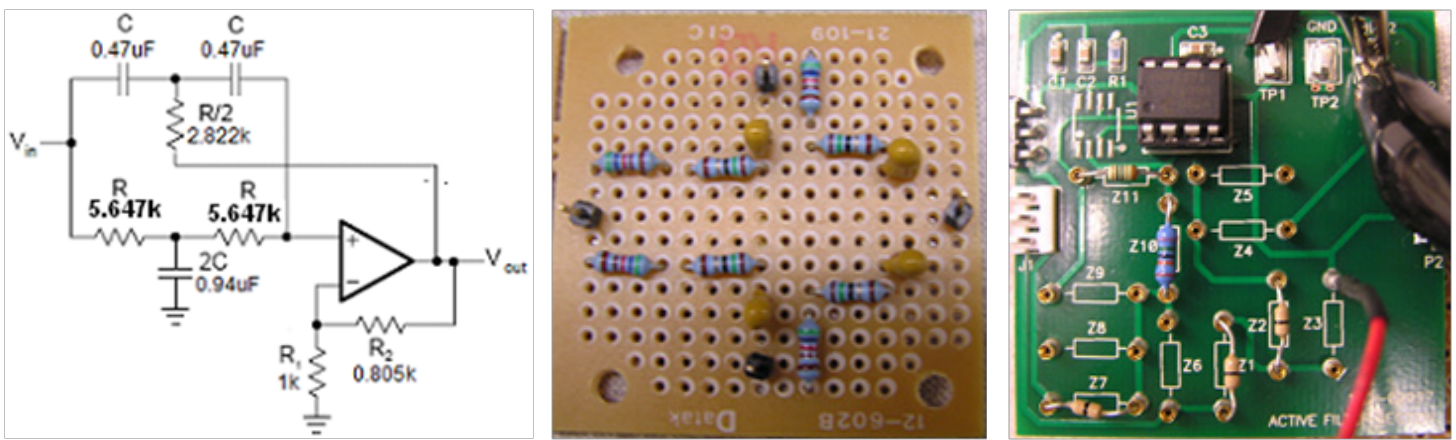

Figure 5 The Twin-T notch filter to remove $60 \mathrm{~Hz}$ powerline noise (A) Notch filter circuit board: passive portion (B) and the active portion (C).

With $f_{n}=60 \mathrm{~Hz}$ and $C=0.47 \mu \mathrm{F}$, the resistor value in the filter is:

$$
R \grave{\mathrm{U}}=\frac{1}{2 \pi f_{n} C}=\frac{1}{2 \pi(60)\left(0.47 \times 10^{-6}\right)}=5.647 \mathrm{k}
$$

Another advantage of the Twin-T configuration is that the quality factor, $Q$, can be altered via the inner gain $G$ without modifying the notch frequency $f_{n}$. To achieve a $Q$ factor of approximately 2.5 , resistances of $R_{1}=1.0 \mathrm{k} \Omega$ and $R_{2}=805 \Omega$ were chosen,

$$
Q=\frac{R_{1}}{2\left(R_{1}-R_{2}\right)}=\frac{1 \times 10^{3}}{2\left(1 \times 10^{3}-805\right)}=2.564
$$

$$
\left\{\begin{array}{l}
C_{21}=4(0.068)=0.272 \mu \mathrm{F} \\
C_{22}=0.068 \mu \mathrm{F}
\end{array}\right.
$$

From equation (8),

$$
\begin{aligned}
& R=\frac{1}{2 \pi C f_{c} \sqrt{m n}}=\dot{\mathrm{U}} \frac{1}{2 \pi\left(0.068 \times 10^{-6}\right)(160) \sqrt{(1)(4)}}=7.318 \mathrm{k} \\
& \left\{\begin{array}{l}
R_{21}=m R=(1)(7.318)=7.318 \mathrm{k} \Omega \\
R_{22}=R=7.318 \mathrm{k} \Omega
\end{array}\right.
\end{aligned}
$$

Similarly, Filter 1 can be designed by letting $f_{c}=160 \mathrm{~Hz}, Q=1$, $n=4.5, m=2, C=0.068 \mu \mathrm{F}$, and calculating $C_{11}=0.068 \mu \mathrm{F}, R_{11}=14.628 \mathrm{k} \Omega$; Filter 3 designed as $C_{31}=0.306 \mu \mathrm{F}, C_{32}=0.068 \mu \mathrm{F}, R_{31}=9.752 \mathrm{k} \Omega$, and $R_{32}=4.876 \mathrm{k} \Omega$. All op-amps in the LPF are MCP6271. This CMOS chip has a $2 \mathrm{MHz}$ Gain Bandwidth Product (GBWP) and a $65^{\circ}$ phase margin. It also supports rail-to-rail input and output swing. ${ }^{19}$

\section{Notch/band-reject filter}

A notch or band-reject filter is typically used in biomedical instrumentation to suppress a certain frequency or range of frequencies in signals. The $60 \mathrm{~Hz}$ power field interference needs to be rejected. This requires a small transition bandwidth or high $Q$ factor to achieve the steeper roll-off. A Twin-T notch filter is one of the few RC networks capable of providing an infinite deep notch at a particular frequency. Two " $T$ " shape RC filters combined with a MCP6271 opamp, as shown in Figure 5A, form an active notch filter. The $Q$ factor can be raised from the usual 0.3 to 2.5 (a Q factor of 50 or more is achievable). Further, the op-amp provides low output impedance and high input impedance, making it possible to use large resistance values in the "T"" so that only small capacitors are required, even at low frequencies. 


\section{Right leg drive circuit}

A right leg drive circuit is used to cancel the common-mode signal between the left and right arm electrodes by inverting, amplifying, and then feeding the signal back to the body through the right leg electrode. As shown in Figure 6, there are three leads connected to the body: a left arm lead, a right arm lead, and a right leg lead. Two AD708 dual op-amps are used: the first one acts as a buffer with a unity gain, and the second one acts as an inverter and amplifier (i.e., inverting amplifier). A gain of 68.19 was achieved with the $866 \mathrm{k} \Omega$ and $12.7 \mathrm{k} \Omega$ gain resistors (i.e., $G=866 / 12.7=68.19$ ). A $0.068 \mu \mathrm{F}$ capacitor in the feedback loop was used to maintain the stability of the right leg drive circuit. The $499 \mathrm{k} \Omega$ resistor at the output end limits the current driven to the body for patient protection. This right leg drive circuit was built on a $3.81 \mathrm{~cm} \times 3.81 \mathrm{~cm}$ prototyping board. The AD8220 requires a $\pm 5 \mathrm{~V}$ DC power supply.

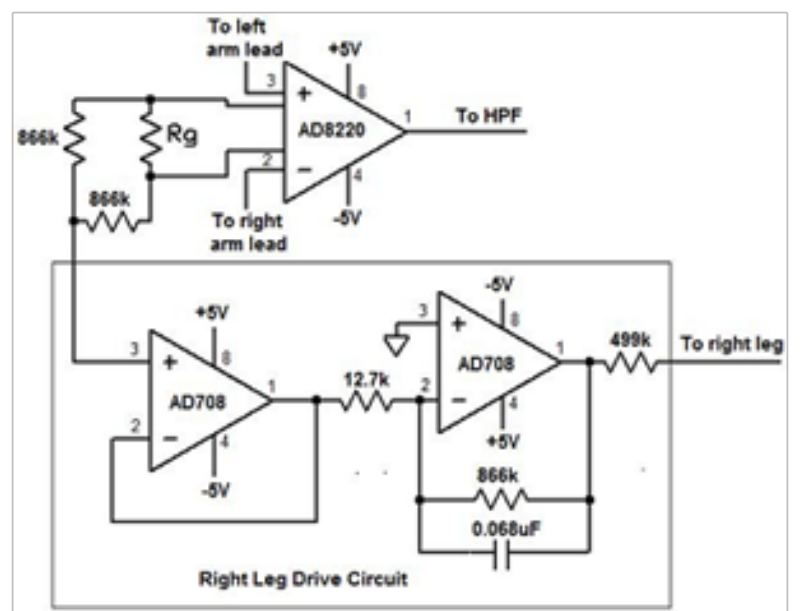

Figure 6 The right leg drive circuit using two AD708 op-amps and one AD8220.

The $\pm 5 \mathrm{~V}$ DC source in the $\mathrm{BC}$ circuit comes from a $\pm 5 \mathrm{~V}$ power supply circuit. It consists of two $9 \mathrm{~V}$ batteries and two voltage regulators. The NTE977 regulates the $+9 \mathrm{~V}$ power supply to $+5 \mathrm{~V}$, while the NTE1917 regulates the $-9 \mathrm{~V}$ supply to $-5 \mathrm{~V}$. The overall ECG signal conditioning circuit is shown in Figure 7. The high-pass, the low-pass, and the notch filters were connected in cascading series. The $\pm 5 \mathrm{~V}$ power supply circuit is located between the right leg drive board and the USB6009. All circuits and evaluation boards, along with the USB 6009 data acquisition device, were mounted on a $40.64 \mathrm{~cm} \times 20.32 \mathrm{~cm}$ base board made of static-dissipative clear cast acrylic sheet. Each circuit or module, as well as their associated components can be easily changed or modified to adapt to different needs for different biosignals.

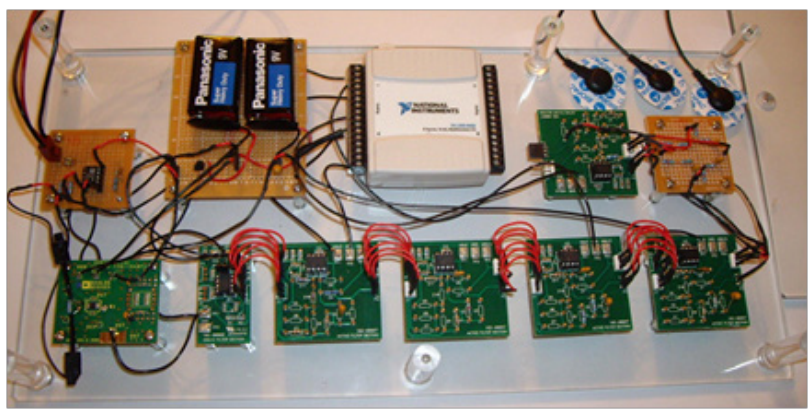

Figure 7 The signal conditioning circuit assembly.

\section{ECG signal testing using the designed BC circuit}

The designed BC circuitry was tested on a real ECG signal from a human subject. The ECG was recorded by the three $\mathrm{Ag}-\mathrm{AgCl}$ electrodes attached to the body and sent to the right leg drive circuit. It then passed through the IA, the HPF, the LPF, and finally the notch filter for a series of conditioning. The ECG output at each stage was recorded, displayed, and examined. In order to examine and display the filtering result at each stage, a USB6009 unit and LabView program ${ }^{20}$ were used for data acquisition, analog to digital conversion, and display. The output of each filter was captured at the $V_{\text {out }}$ pin located at the top edge of each filter board and was connected to one of the terminals on the USB6009 unit. Figure 8 shows the ECG outputs at each filtering stage. The instrumentation amplifier first amplified the original ECG signal to raise its voltage level (with a gain of 19). At this stage, no visible ECG peaks were observed. After the high-pass filter and further amplification, the "bump" features of the ECG signal started to appear, although there was high frequency noise riding on the ECG output. The subsequent low-pass filter attenuated the high frequency component. However, $60 \mathrm{~Hz}$ power line interference was still visible. At this stage, the ECG peaks - $P, R, S, T$ - became more distinct. The notch filter was able to remove the power line noise, resulting in a very clear and smooth ECG signal with $P, Q, R, S$, $T$ peaks even more distinguishable. This final output of the ECG waveform suggested that the designed ECG conditioning circuit functioned properly and the results are comparable with commercial products.

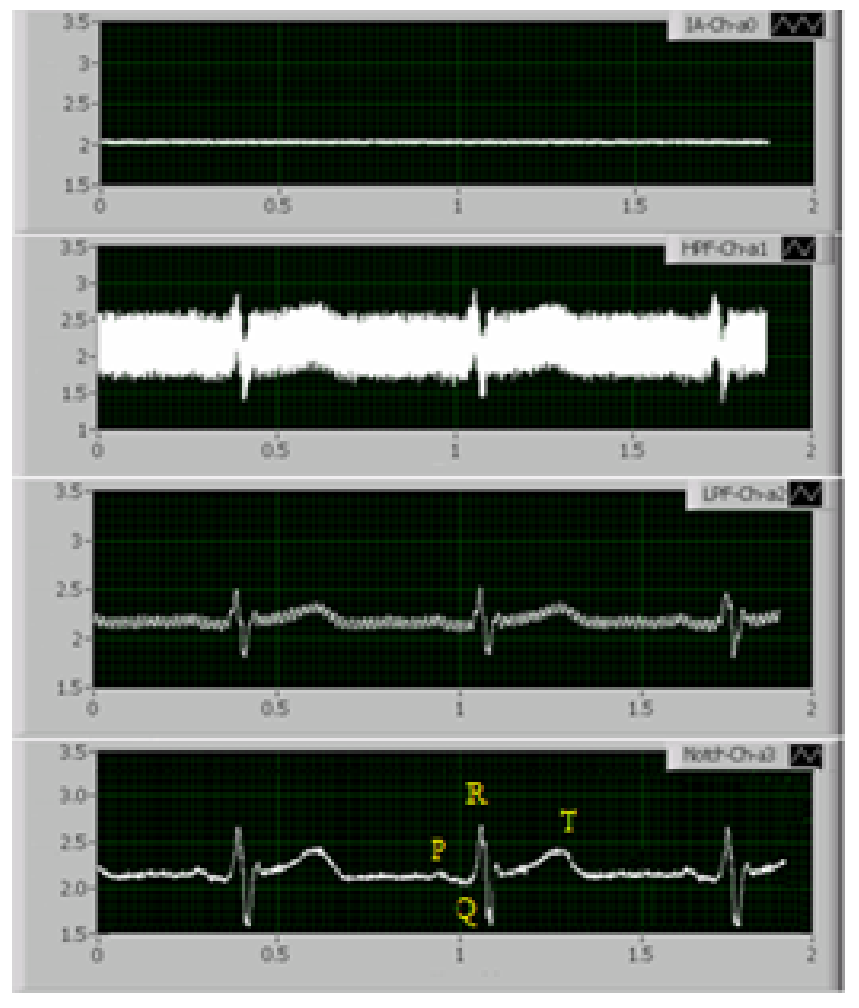

Figure 8 ECG outputs at each stage of signal conditioning- IA, HPF, LPF, and notch filter.

\section{Conclusion}

The sequential usage of the IA, the HPF, the LPF, and the notch filters have resulted a successfully conditioning a raw ECG wave from 
a human subject. The merits of this circuity not only lie its excellent functionality, but also its flexible modularity. Each filter unit, as well as their associated components, can be easily changed or modified to adapt to other biosignals with different amplitudes and frequencies. The system could also be used to condition other non-biosignals. The device provides a useful educational platform for students to learn, step-by-step, signal conditioning principles, function and construction of active and passive filters, electronic components, interface, and LabVIEW programming tools.

\section{Acknowledgements}

None.

\section{Conflict of interest}

The author declares no conflict of interest

\section{References}

1. Global, regional, and national age-sex specific all-cause and causespecific mortality for 240 causes of death, 1990-2013: a systematic analysis for the global burden of disease study 2013. Lancet. 2015;385(9963):117-171.

2. Provaznik I. Adaptive systems in ECG processing. Czech Republic: Technical University of Brno; 1995.

3. Tenedero CM, Raya MA, Sison LG. Design and implementation of a single-channel ECG amplifier with DSP post-processing MATLAB. Instrumentation, Robotics, and Controls Laboratory, USA: University of Philippines; 2017.

4. Fulford-Jones T, Wei G, Welsh M. A portable, low-power, wireless twolead EKG system. Enginnering in Medical and Biology Society (EMBS). 2004. p. 2141-2144.

5. Matviyehko S. Low-cost EKG pulsometer. Cypress Semiconductor Corp, USA: Springer; 2006.

6. Raju M, Heart R. EKG monitor using the MSP430FG439. USA: Texas Instrument Inc; 2005.
7. Company-Bosch E, Hartmann E. ECG front end design is simplified with microconverter ${ }^{\circledR}$. USA: Analog Devices, Inc; 2005.

8. Seydnejad SR, Kitney RI. Real-time heart rate variability extraction using the Kaiser window. IEEE Trans Biomed Eng. 1997;44(10):990-1005.

9. Blackburn NA. Enhancing ECG quality: update on site selection, skin preparation, and electrode placement. Conmed Corporation, USA: 2003.

10. Carr JJ, Brown JM. Introduction to Biomedical Equipment Technology, 3rd ed. USA: Prentice Hall Inc; 1998.

11. Hosseini H, Nazeran H, Reynolds KJ. ECG noise cancellation using digital filters. Proceedings of the $2^{\text {nd }}$ International Conference on Bioelectromagnetism; Australia: IEEE; 1998. p. 151-152.

12. Bronzino JD. The Biomedical Engineering Handbook. 3rd ed. Medical devices and systems, USA: CRC Press Inc; 1995.

13. Malmivuo J, Plonsey R. Bioelectromagnetism-principles and applications of bioelectric and biomagnetic Fields. USA: Oxford University Press; 1995.

14. G Wolfe, The professional biomed on ECG troubleshooting 101.

15. JFET input instrumentation amplifier with rail-to-rail output in MSOP package. USA: Analog Devices Inc; 2006-2010.

16. Count L, Kitchin C. A designer's guide to instrumentation amplifiers. 2nd ed. USA: Analog Devices Inc; 2004.

17. Rijnbeek PR, Kors JA, Witsenburg M. Minimum bandwidth requirements for recording of pediatric electrocardiograms. Circulation. 2001;104(25):3087-3090.

18. J Karki. Active lows pass filter design, Application report. USA: Texas Instrument Inc; 2002.

19. MCP6271/2/3/4/5. Microchip Technology Inc, USA: Springer; 2004.

20. Chang C, Han J, Ramjeet D. LabVIEW ${ }^{\mathrm{TM}}$ design of a vectorcardiograph and 12-lead ECG monitor. Report submitted to department of electrical and computer engineering. Canada: University of Manitoba; 2003. 